Pérégrinations d'une analyste du discours en territoire éthique : la prise de position dans tous ses états

Peregrinations of a Discourse Analyst in the Field of Ethics: The Ins and Outs of Taking a Stand

\title{
Roselyne Koren
}

\section{(2) OpenEdition}

\section{Journals}

Édition électronique

URL : http://journals.openedition.org/pratiques/2358

DOI : 10.4000/pratiques.2358

ISSN : 2425-2042

Éditeur

Centre de recherche sur les médiations (CREM)

Édition imprimée

Date de publication : 31 décembre 2014

Référence électronique

Roselyne Koren, « Pérégrinations d'une analyste du discours en territoire éthique : la prise de position dans tous ses états ", Pratiques [En ligne], 163-164 | 2014, mis en ligne le 31 décembre 2014, consulté le 02 mai 2019. URL : http://journals.openedition.org/pratiques/2358; DOI : 10.4000/pratiques.2358

Ce document a été généré automatiquement le 2 mai 2019.

(c) Tous droits réservés 


\section{Pérégrinations d'une analyste du discours en territoire éthique : la prise de position dans tous ses états}

Peregrinations of a Discourse Analyst in the Field of Ethics: The Ins and Outs of Taking a Stand

Roselyne Koren

1 Cette contribution a pour objet la reconstitution d'un parcours épistémologique personnel. Il s'agit d'y présenter et d'y justifier les pérégrinations d'une linguiste, analyste du discours et de l'argumentation, en territoire éthique. Je voudrais préciser toutefois d'emblée, avec M. Doury (2013: § 4), que la décision de prendre mon propre parcours pour objet n'est nullement à interpréter comme «l'indice [...] d'un narcissisme surdimensionné ». Ma contribution souhaite uniquement rendre compte des voies suivies pour tenter de réaliser un objectif épistémique semblable, en dépit de différences épistémologiques consistantes, à celui que J.-P. Desclés (2009: 51) définit dans Langue française 162, numéro consacré à "la prise en charge » comme "une linguistique qui cherche à accroître sa scientificité par une inquiétude épistémologique continuelle pour une meilleure compréhension et critique des concepts qu'elle utilise et construit » (je souligne).

2 Ces précautions étant prises, je m'engage dans la voie d'un questionnement éthique ancré dans l'observation de pratiques discursives et argumentatives, mais également dans un cadre théorique interdisciplinaire permettant de combler des manques épistémiques. La décision de recourir à ce cadre est due au constat suivant: les théories linguistiques et l'analyse du discours contemporaines, en France, marginalisent ou passent sous silence un domaine pourtant socialement primordial : le champ de l'évaluation axiologique et du jugement de valeur. Les travaux pionniers de C. Kerbrat-Orecchioni sur la subjectivité axiologique dans le langage (1980) et la mauvaise foi argumentative $(1981,1986)$ constituent une exception dans ce paysage scientifique. 
3 Par questionnement éthique j'entends tout d'abord la recherche des lieux discursifs où le sujet du discours évoque et argumente ce qui fait sens pour lui, les valeurs qui le stimulent, construisent son identité et guident ses actes de parole, lieux où il peut aussi énoncer ses dilemmes existentiels et où il justifie ses choix face à l'Autre du discours. La prise en compte de l'altérité est en effet l'un des traits intrinsèques de tout questionnement éthique. L'allocutaire joue alors un rôle essentiel : il remplit une fonction critique ; la rationalité des dires de l'un dépend certes tout d'abord de la justification de ses arguments, mais aussi de l'assentiment de l'Autre, son alter ego, mais aussi son juge.

4 Ce questionnement éthique est aussi celui du chercheur, analyste de corpus authentiques où il peut $\mathrm{y}$ avoir manipulation de l'opinion d'autrui, mauvaise foi argumentative, diabolisation, incitation à la haine ou à la discrimination de l'Autre, argumentation idéologique mortifère, dénégation de toute forme de responsabilité. Il s'agira alors de justifier mes réponses aux questions suivantes : le chercheur se doit-il de rester neutre en permanence, quel que soit son corpus, ou est-il possible et même parfois nécessaire de franchir la ligne rouge entre neutralité et prise de position évaluative ? Bref, la neutralité absolue est-elle valide en permanence? Garantit-elle automatiquement l'accès à la totalité du savoir à construire? L'éthique du chercheur a donc ici tout d'abord la réflexivité pour condition de possibilité ; rationalité et autocritique sont alors, comme l'affirme O. Reboul (1980: 197), étroitement liées.

Le parcours épistémique que je vais tenter de reconstituer a pour origine la décision d'explorer «les espaces discursifs» que C. Kerbrat-Orecchioni (1981: 63) qualifie de «marécages » du fait qu'« il est bien difficile d'opérer des tracés de frontière (entre le normal et le déviant, le légal et le fautif, l'honnête et le malhonnête) ». Mais elle ajoute : "Si les incertitudes du langage » et "le caractère précaire et fluctuant de la norme argumentative " sont « responsables de ces difficultés, elles fondent en même temps la possibilité d'exercice de la mauvaise - donc de la bonne - foi ». Ce parcours comprendra les étapes suivantes ${ }^{1}$ : problématisation de la question de l'objectivité et donc de la prise de position dans le langage, mise en question de l'identité du sujet du discours puis exploration de la prise en charge du vrai référentiel et du juste ou du bien; proposition de réajustement de l'éthique du chercheur en sciences du langage. Ce qui relie ces diverses questions entre elles, c'est leur successivité chronologique, mais surtout le fait que chacune d'elles se définit par rapport à un acte commun : l'acte de juger entendu ici comme évaluer, hiérarchiser, trancher et justifier et non pas comme dresser un réquisitoire ; or cet acte est perçu et présenté, dans la majorité des discours épistémiques en sciences du langage en France, comme un acte suspect, comme une menace pour une éthique souhaitant accorder une place primordiale à un "rationalisme égalitaire " (Perelman, 1989 : 202). Derrière la décision de se prononcer pour ou contre, de valoriser ou de dévaloriser, bref de prendre clairement position - décision dont la rationalité n'irait pas de soi-, se profileraient presque automatiquement les spectres de l'esprit partisan, de la partialité, de l'idéologie militante et de la prescription moralisatrice. Tout se passe comme si le fait même de prendre la prise de position axiologique pour objet risquait de compromettre la scientificité et la rationalité de la recherche. On essaiera ici au contraire de justifier la décision de problématiser et de réduire cette défiance et de démontrer que l'acte de juger peut être un acte rationnel aussi valide et vital que celui d'informer et de représenter les réalités de l'environnement telles quelles. En résumé, l'enjeu épistémique de ces pérégrinations est le parcours d'une recherche ayant tenté de revisiter la question de la rationalité et de la légitimité du jugement de valeur et de ses 
interactions avec le jugement de fait ou de vérité dans le discours et l'argumentation rhétorique.

6 Ce parcours est donc ancré dans les tentatives effectuées afin de comprendre et d'éclairer les paradoxes suivants : l'évaluation, l'éloge, le blâme, l'idéalisation ou la diabolisation, les discours polémiques récurrents occupent une place centrale dans les espaces publics comme dans les cadres privés des interactions verbales; la théorie des faces nous enseigne que l'homme est un loup pour l'homme et que les difficultés de la communication intersubjective seraient comparables à celles que rencontre le funambule (j'emprunte cette métaphore à Kerbrat-Orecchioni, 2005). Pourtant, dans la plupart des cas, les théories du langage problématisent uniquement en France la prise en charge consensuelle du vrai référentiel. L'évaluation axiologique est laissée dans l'ombre ou évoquée subrepticement et frileusement. Autre paradoxe : le locuteur peut qualifier un «miroir» de «virulent » ou mêler lexèmes axiologiques et tournures impersonnelles dans le même énoncé, présenter un jugement de valeur sous les apparences irréfutables de l'évidence comme dans «c'est vrai, mal, c'est bien» ou encore discréditer une cible politique en la qualifiant de « nazisme tropical (J.-P. Chrétien, Libération, 26/04/94), sans que la plupart des linguistes français n'en déduise qu'il faille mettre en question la suprématie de la thèse de la description neutre, objective et spéculaire d'un langage à visée essentiellement informationnelle et vériconditionnelle.

7 C'est à la croisée interdisciplinaire de l'analyse du discours, de la nouvelle rhétorique perelmanienne, de la philosophie et de la sociologie que j'ai trouvé des hypothèses explicatives me permettant de tenter de résoudre ces paradoxes sans jamais perdre de vue le système du langage et du discours. C'est à des auteurs comme C. Perelman, philosophe du droit, logicien et rhétoricien, aux philosophes H. Arendt, R. Polin, R. Rorty, $\mathrm{O}$. Reboul, aux sociologues $\mathrm{M}$. Weber et R. Boudon que je dois les hypothèses explicatives m'ayant permis de comprendre et de surmonter les silences épistémiques de ma discipline sur la question de la mise en mots des valeurs et de la prise de position. Je donnerai cependant ici la primauté à la nouvelle rhétorique perelmanienne (désormais NR) pour ne pas dépasser la place qui m'est impartie. La NR a été en effet déterminante pour moi dans la mesure où elle ancre sa conception de l'argumentation dans les pratiques discursives de corpus authentiques, spécifiques des espaces discursifs de la vie politique et sociale.

\section{«Ces pommes ne me disent rien »/ « je n'aime pas ces pommes »(Perelman, $1983: 243)^{2}:$ de l'objectivité dans le discours}

C'est dans le cadre d'un cours de rhétorique figurale et argumentative destiné à de futurs traducteurs que la centralité de la problématique de l'impartialité et de l'objectivité m'est apparue. On m'avait en effet demandé de ne pas prendre comme objet des textes littéraires, mais d'analyser de préférence des articles de presse écrite en français. Il m'est vite apparu que le couple apparences/réalité - neutralité apparente/point de vue subjectif relégué dans l'implicite - jouait un rôle central dans la mise en mots de l'information. Les énoncés suivants de type "ça parle », énoncés par un médiateur absent de ses propres dires, étaient omniprésents quel que soit le genre d'article : «Mais déjà s'impose une de ces évidences qui fondent les meilleures ententes", "l'élection de 
Bordeaux ne saurait être présentée comme un test national ", «l'élection traduit une attitude qui dément... » (Koren, 1996: 80-81). Le devoir d'objectivité occupait également une place centrale dans les métadiscours des journalistes sur la déontologie de la profession. La plupart d'entre eux y affirmaient leur désir d'être la voix-off, celle de la transmission spéculaire et «filmique » de la parole des faits. Mais ils souhaitaient aussi simultanément - là résidait le paradoxe - éclairer les lecteurs, les libérer de l'emprise des institutions politiques et donc pratiquer à l'égard de ces dernières un "devoir d'irrespect » - soit proposer des analyses critiques nécessairement évaluatives du pouvoir exécutif (Koren, 1996 : 143-170).

9 La compatibilité de ces deux enjeux contraires ne pouvait pas être pensée dans le cadre de théories du langage informationnelles et vériconditionnelles: d'où venait donc ce puissant désir d'effacement énonciatif ? Pourquoi l'énonciation de « virulent » devait-elle être «rachetée » (Rastier, 1994 cité in: Koren, 1997a : 163) par celle de «miroir » et la dénonciation d'« une détestable mauvaise foi » par la tournure impersonnelle à la fois prescriptive et euphémisée - «il faudrait donc être (d'une détestable mauvaise foi)»? Pourquoi ce désir de "dire » sans avoir l'air de dire (Ducrot, 1972) ? Quelle était donc cette doxa qui exigeait que le locuteur relègue ses prises de position évaluatives dans le non-dit? Pourquoi cherchait-on à donner à l'opinion les apparences de l'évidence ?

10 La lecture du Traité de l'argumentation de C. Perelman et L. Olbrechts-Tyteca (1983) et la NR qui y est théorisée m'ont permis d'élaborer les hypothèses explicatives nécessaires à ces recherches et de tenter de combler le vide épistémique évoqué ci-dessus. La NR est certes ancrée dans une réflexion philosophique, mais elle est proche des sciences du langage puisque $\mathrm{C}$. Perelman se refuse à dissocier la forme du fond: il insiste sur le fait que sa théorie de l'argumentation, qui est aussi une « logique des jugements de valeur », a pour objet les «moyens discursifs d'obtenir l'adhésion des esprits » (Perelman, $1983: 10)$. Son corpus privilégié se veut, comme il le précise (ibid.: 13) constitué par les argumentaires de journalistes, de politiciens, d'avocats, de juges et de philosophes.

11 J'ai eu le sentiment, dès les premières lignes du Traité de l'argumentation, d'avoir trouvé une hypothèse explicative fondationnelle. On y lit effectivement :

«La publication d'un traité consacré à l'argumentation et son rattachement à une vieille tradition, celle de la rhétorique et de la dialectique grecques, constituent une rupture avec une conception de la raison et du raisonnement issue de Descartes, qui a marqué de son sceau la philosophie occidentale des trois derniers siècles » (ibid.:1).

Cette "rupture » a la validation et la légitimation du vraisemblable et donc de l'opinion pour enjeu. Il s'agit de désacraliser la toute-puissance des thèses suivantes : le seul régime de rationalité valide est celui d'un sujet pensant que sa raison rend apte à percevoir, à contempler et à incorporer des vérités préétablies irréfutables. Le désaccord ne peut plus être de ce fait qu' " un signe d'erreur» (ibid.: 2) ; le raisonnement "more geometrico» constituerait le modèle unique et universel sur lequel construire le savoir et un discours garantissant la valeur par excellence : la vérité référentielle objective. Or, C. Perelman est convaincu que cette "limitation" est non seulement indue, mais qu'elle prône une dichotomie inacceptable entre les facultés de l'esprit humain, qu'il refuse de hiérarchiser ${ }^{3}$ . Comme on le verra plus bas, s'opposer à la conception de la raison théorique défendue par R. Descartes, c'est argumenter en faveur de la complémentarité de régimes de rationalité foncièrement différents, mais parfaitement conciliables dans le discours, mettant en œuvre l'aptitude de l'homme à formuler des jugements de vérité et des jugements de valeur; c'est problématiser la question de l'identité du locuteur. Celui-ci 
n'est plus perçu dans la NR comme un "sujet pensant ", mais comme une "personne ", un sujet d'énonciation partiellement autonome qui argumente des choix éthiques dont il se considère comme responsable. La liberté de ces choix n'est pas limitée par l'obligation de s'aligner sur un modèle logique rationaliste, mais par le regard critique de l'Autre, son alter ego qu'il doit persuader de la légitimité de ses dires.

Les auteurs du Traité décrivent, d'une part, les procédures ayant pour enjeu de donner à des jugements de valeur les apparences du jugement de fait et l'autorité de l'évidence : ils désignent ainsi quelques observables emblématiques étroitement liés à une conception cartésienne de la raison. Mais on verra aussi ci-dessous que la controverse ne se limite pas à ce travail de dévoilement ; la NR revisite la définition de l'objectivité dans le discours et l'argumentation. Il s'agit en fait dans le premier cas de tenter de neutraliser à priori les vélléités de réfutation de l'auditoire. Le recensement de ces procédures a joué un rôle déterminant dans les chapitres de R. Koren (1996: 21-134) consacrés à l'objectivité de l'écriture de presse. On compte parmi ces procédures: le mythe de la parole des faits, du " ça parle », simplement, mais très clairement défini par les deux énoncés insérés dans le titre ci-dessus. La différence entre ces deux énoncés (ibid. : 243) serait de l'ordre suivant: « je n'aime pas ces pommes » est un aveu subjectif assumé explicite, « ces pommes ne me disent rien » transforme un « jugement de valeur » en « jugement de fait ». « On reproche à l'objet, affirment C. Perelman et L. Olbrechts-Tyteca (1983), de ne pas adresser d'appel, on considère que si l'on réagit défavorablement cela résulte d'un comportement de l'objet", ce qui exonère le sujet d'énonciation de toute responsabilité. Un terme "neutre » est en fait aux yeux du théoricien de la NR un terme susceptible de passer « inaperçu » et qui créerait l'illusion d'un «langage descriptif en soi » (ibid. : 202), sur lequel « l'aspect social du langage, instrument de communication et d'action sur autrui » n'aurait pas prise. Il serait possible pour un tenant de l'objectivité absolue de comprendre le sens configuré par ce type de langage sans tenir compte du « contexte fourni par les habitudes, les façons de penser, les méthodes, les circonstances extérieures et les traditions connues des usagers" (ibid.: 680-681). Le pronom indéfini on suffit à transformer le sujet en médiateur anonyme au service de la collectivité; le locuteur tente ainsi de transformer "le subjectif " " en normal » afin de le soustraire à la réfutation, diminuant aussi, du même coup, « la responsabilité dans le jugement » (ibid. : 218) ${ }^{4}$. La NR démontre enfin que ce langage prétendument descriptif feint d'ignorer le lien qui lie la description au «but » qu'elle « poursuit » et la «pensée » à l'« action» (ibid. : 208), lien particulièrement crucial dans le domaine de l'information et de la transmission d'un savoir permettant d'agir en connaissance de cause dans la vie politique et sociale.

Les techniques recensées ci-dessus problématisaient la tentative de donner de force au vraisemblable les apparences de l'évidence. Derrière les titres d'éditoriaux du type «Le poids des faits» ou les énoncés "la crise exige ", «la situation traduit », «l'ironie du destin a voulu que » allaient se profiler dorénavant pour moi le refus de reconnaitre la subjectivité inéluctable du regard individuel ou la décision de le faire passer « inaperçu » ou de lui donner les apparences d'une vérité consensuelle. Je ne pourrai plus lire désormais un énoncé comme " la crise exige » ou " c'était une évidence déjà c'est un fait établi désormais» (Koren, 2004b) sans y voir ce que la sacralisation de la vérité référentielle fait au discours : favoriser la tentative de domination de l'Autre, la volonté d'avoir raison de son éventuelle résistance au lieu de tenter de négocier la distance qui les sépare. 
La NR m'a également permis, d'autre part, de revisiter la question de la définition de la notion de "fait» dans la vie politique et sociale ainsi que la distinction entre « impartialité » et « objectivité ». Le " poids des faits », argument particulièrement cher aux journalistes réalistes ou aux positivistes qui y voient un argument apte à discréditer à priori toute velléité d'indépendance ou de contestation, y est déstabilisé par l'argumentation suivante : les auteurs du Traité (Perelman, Olbrechts-Tyteca, 1983 : 89-90) se refusent purement et simplement à donner une définition intrinsèque de la notion de fait. Celui-ci n'aurait pas d'existence à priori dans le champ de l'argumentation; il prendrait corps dans la discussion entre argumentateurs s'ils parviennent à négocier un accord. Aucun fait ne peut être asserté de façon absolue s'il est contesté par une partie de l'auditoire. C'est par ailleurs dans un développement intitulé "Argumentation et engagement » (ibid. : 78-83) que C. Perelman et L. Olbrechts-Tyteca proposent de revisiter la question de la neutralité et de distinguer entre « impartialité » et « objectivité » afin de définir la fonction du médiateur invité à juger les acteurs d'un conflit. La qualité majeure du tiers médiateur n'est pas à leurs yeux l'objectivité, car cela signifierait qu'il ne partage pas les valeurs de la collectivité qui l'a choisi et n'en est pas «solidaire». Il se doit par contre d'être impartial lors d'un recensement initial, heuristique et pluraliste des points de vue. Il doit y avoir alors et seulement alors suspension de toute forme de jugement - soit «impartialité ». Ce dernier revient cependant en force à l'étape suivante où le médiateur se doit de trancher dans le cadre d'une éthique de responsabilité où l'action est une valeur fondamentale. Les questions de l'objectivité et de la subjectivité ne peuvent plus être pensées de ce fait au prisme du rationalisme cartésien et du modèle épistémique de la démonstration scientifique; elles doivent être «repensées » et "réinterprétées » "pour qu'elles puissent avoir un sens dans une conception "pratique" de l'argumentation qui «se refuse à séparer une affirmation, de la personne de celui qui la pose » (78) et en assume la responsabilité énonciative.

16 La critique perelmanienne de la raison théorique cartésienne et de la conception de la connaissance qui en découle m'a enfin permis de comprendre pourquoi l'objectivité absolue est sémiotisée dans l'écriture de presse et dans tout autre discours où elle est mise en œuvre, par un mouvement de balancier soit par l'oscillation binaire descriptive entre deux pôles antithétiques : le pour et le contre, le noir et le blanc, l'apologie sur la page de gauche et la dénonciation sur celle de droite (voir Koren, $2001: 181$ ). Il existerait un rapport de cause à effet entre la conviction que l'accès à la vérité référentielle dépend de la contemplation collective d'évidences existant à priori et la délégitimation du jugement de valeur, perçu comme un obstacle entravant la réalisation de ce type de consensus. L'oscillation «rationaliste» " égalitaire» (Perelman 1989: 202) entre un discours et son contrediscours constituerait de ce fait le degré ultime de l'équité. Ce raisonnement m'a permis de comprendre pourquoi le terroriste pouvait être présenté dans le même article, simultanément, comme un "résistant » héroïque et un criminel (Koren, 1996: 259-267 ; 2006a : 101-104) et donc donner lieu à des actes de nomination antithétiques concomitants. Il m'a aussi permis de comprendre cet extrait paradoxal du numéro spécial consacré par Libération à la commémoration de ses 20 ans (1) où la systématisation rhétorique de l'oscillation atteint un paroxysme et cette analyse de J.-F. Kahn concernant l'oscillation médiatique binaire en général (2):

(1) «Prendre une période donnée [...] et se l'approprier, ne serait-ce qu'au nom de l'arrachement [...] Manuel des civilités binaires à l'usage des jeunes générations ? [... ] On n'hésitera pas une seconde [...] à transgresser sciemment cette règle de fer à peine édictée. " 
(2) «La pensée bipolaire ne secrète plus qu'un discours de défense de la bipolarité

elle-même » (Koren, 2001 : 178, 193). journalisme mis au net ? Il semble que oui : celle de cet oxymore, par exemple, définissant le credo du Canard enchaîné (Koren, 1996: 267) : l'« anarchisme cartésien ». La figure ne réfère pas à une opposition dichotomique aporétique, mais sémiotise une interaction féconde entre la liberté de penser et de juger, le refus de toute forme de domination et une conception rigoureuse vériconditionnelle de l'information.

\section{Le « sujet » au prisme d'un questionnement éthique}

La notion de sujet est la condition de possibilité fondationnelle de tout questionnement éthique; celui-ci constitue avec l'Autre, son alter ego, un couple notionnel incontournable. L'analyse du discours (désormais $\mathrm{AD}$ ) accorde certes une place centrale à la notion de sujet, mais semble plus intéressée par les contraintes qu'il a à subir et celles qu'il cherche à faire peser sur l'énonciataire que par leurs libertés respectives (Koren, 2008b, à paraître). D. Maingueneau (1999: 79-80) le présente comme un être de langage, essentiellement garant et responsable de la vérité référentielle de ses dires, construisant sa " manière d'être » par le biais d'une « manière de dire »; P. Charaudeau et lui (2002: 224-228) soulignent la nature coercitive des rapports intersubjectifs. Mais peut-il en être autrement lorsque la valeur par excellence, celle qui sous-tend la réflexion sur la responsabilité du locuteur se limite à la vérité référentielle ou que l'analyste du discours s'interroge, tout au plus, sur la responsabilité du locuteur au prisme de la polyphonie ? Le sujet de l'argumentation et donc de l'éthique du discours prônée par la NR est un être de langage partiellement autonome, responsable de ses jugements de fait comme de ses jugements de valeur. Il accorde une importance égale au vrai, au juste ou au bien. Le sujet de l'AD est certes moins désincarné que le locuteur des théories linguistiques: son discours est indissociable de la situation d'énonciation sociohistorique où il prend la parole, mais il est soumis aux doxas et aux contraintes extérieures de systèmes de valeurs inhérents aux genres discursifs ou aux scénographies auxquels il recourt. Le carcan de l'argumentaire du sujet de l'argumentation est avant tout son auditoire : c'est à ce dernier qu'il incombe de juger les justifications mises en œuvre afin de légitimer ce qui fait sens pour l'argumentateur, de se prononcer sur leur cohérence et leur rectitude et de partager la responsabilité de la rationalisation des prises de position.

Je dois donc à cette conception perelmanienne de l'autonomie du sujet éthique le cadre théorique m'ayant permis de penser la responsabilité énonciative et/ou collective qu'implique, par exemple, l'acte de nommer le terroriste ou l'attentat (Koren, 1996, 2006a, 2008a). La conception du sujet de l'argumentation défendue par la NR défend en fait la même thèse que celle défendue par le sociologue R. Boudon (1995) dans Le juste et le vrai : il existe un régime de rationalité des jugements de valeurs aussi «robuste» ( j'emprunte ce qualificatif à Paveau, 2013) que celui des jugements de fait des sciences exactes; le sujet est parfaitement capable, à ses yeux, de justifier clairement, consciemment et rigoureusement ses jugements de valeur ; la justification en garantirait la rationalité et la validité.

Cette conception du sujet autonome et de ce fait responsable de ses mises en mots, en dépit de multiples contraintes discursives, institutionnelles et sociales, sujet indissociable de son alter ego comme le « je » et le « tu » de la linguistique énonciative d'É. Benveniste, 
m'a permis de comprendre la révolution rhétorique effectuée dans l'écriture de presse mise au net: le retour en force d'une subjectivité décomplexée, la revalorisation et la déculpabilisation de l'acte de juger et de prendre position. De longues années de sacralisation de l'objectivité, la dénégation de la responsabilité énonciative, la passivité de l'auditoire, la posture en surplomb du journaliste, porte-parole de l'opinion publique, plus proche des institutions que de ses lecteurs ont joué et jouent toujours encore un rôle central dans le conflit de légitimité entre "anciens » et "nouveaux » médias (Koren, 2013a).

\section{"Une conception unificatrice de la rationalité » (Ribeiro, 2012 : 176, 183) : la prise en charge du vrai et du juste}

21 La NR n'est certes pas une théorie du langage, ni C. Perelman un linguiste, mais la thèse de l'intrication et des interactions du jugement de fait et du jugement de valeur constitue, à mes yeux, une contribution méconnue à l'analyse de l'hétérogénéité du langage et du discours et des fonctions argumentatives du métadiscours. Elle ouvre la voie à un rapport éthique aux fonctions à la fois représentationnelles et évaluatives du langage soit à la prise en charge concomitante du faire croire vrai et du juste (entendu comme équitable) ou du bien et de leurs contraires. Il devient possible de penser que la raison théorique n'est pas le seul mode d'élaboration et de configuration du concept de rationalité et que, comme le souligne R. Boudon (1995: 541 ), serait rationnel tout énoncé quel qu'il soit intégrant la justification du point de vue défendu : « $X$ avait des raisons de croire (faire) $Y$, car... » et « irrationnelles » les explications introduites par un raisonnement du type : « $\mathrm{X}$ n'avait pas de raisons de croire (de faire) $Y$, mais... ", soit $X$ affirme $Y$, bien qu'il soit incapable d'en justifier le bien-fondé, $Y$ est une affirmation gratuite, non valide. Les auteurs du Traité de l'argumentation (1983: 681-682) affirment ainsi : «La pratique et la théorie de l'argumentation sont, à nos yeux, corrélatives d'un rationalisme critique, qui transcende la dualité jugements de réalité-jugements de valeur, et rend les uns comme les autres solidaires de la personnalité du savant ou du philosophe, responsable de ses décisions dans le domaine de la connaissance comme dans celui de l'action.» L'unification des deux catégories de prise de position a la conscience éthique du sujet pour condition de possibilité : celui-ci peut parfaitement faire preuve, comme le souligne E. Eggs (1999: 43) d'« intégrité discursive » soit d'un rapport «neutre », « objectif» ou «stratégique» au réel référentiel et avoir alors la véridiction pour norme et, simultanément, faire preuve d'« intégrité rhétorique » soit d'un rapport justifié à des valeurs autres que le vrai et avoir simultanément la rectitude éthique pour norme. C'est alors par le biais de la justification jugée et évaluée par l'Autre du discours que l'éthique et la rationalité axiologique viennent (ou non, si dissensus il y a) au discours. Le système du langage confirme en l'occurrence la pertinence des prises de position philosophiques, éthiques ou rhétoriques contestant la thèse d'une oscillation dichotomique entre fait et valeur, mais aussi celle de la sacralisation de la raison théorique, de «l'homme spéculaire " reflétant les réalités extérieures telles quelles et de l'inexistence d'un régime de rationalité axiologique dans le langage.

Ce qui fait donc, à mes yeux, de la NR une éthique du discours, c'est le refus de considérer qu'il existe des normes morales indiscutables et une vérité $\mathrm{V}$ atemporelles à priori, c'est 
aussi la conviction que dans l'univers du vraisemblable et des interactions verbales, qui est celui de la société civile, l'objectivité absolue n'existe pas. Les faits, les vérités et les valeurs y sont discutables et nécessairement discutés et négociés par le sujet d'énonciation et son énonciataire. Les coénonciateurs dominés de l'AD y sont transformés en êtres de langage dont l'autonomie n'a de sens que parce qu'ils sont à la fois libres, responsables et dépendants du jugement d'autrui. Et par liberté, la NR entend liberté de prendre position, de juger et de se transformer (Perleman, Olbrechts-Tyteca, 1983 : 397), liberté de choisir et de configurer l'« auditoire universel » soit le modèle exemplaire intérieur de la rationalité dont ils tentent de se rapprocher sans jamais pouvoir y parvenir ${ }^{5}$. L'aspiration éthique au dépassement de soi est en effet un trait intrinsèque du sujet de l'argumentation; on la perçoit dans la gestion des justifications, mais aussi dans la rhétorique figurale et argumentative de discours épidictiques (Koren, 2010a; 2015) où le sujet est animé comme le souligne D. Jamet (2008: 11) par «la force de paroles qu'anime un souffle", " il y a des mots, ajoute-t-il, qui portent celui qui les prononce et ceux qui les entendent au-dessus et au-delà d'eux-mêmes » (je souligne).

\section{De la théorie à la pratique}

Le moment est venu de mettre brièvement ce parcours à l'épreuve de l'analyse de pratiques argumentatives et discursives ${ }^{6}$. Je me limite à l'analyse de quelques extraits d'un exemple type publié sur le net par l'association Acrimed (Action Critique Médias) afin de ne pas dépasser les limites de l'article. L'analyse critique, au ras des mots, de discours médiatiques au service des institutions politiques est l'une des activités majeures de cette association de la société civile. Ce texte de cinq pages s'intitule « Fort avec le faible, faible avec le fort: l'anticonformisme de la revue Médias » et problématise la question de «la liberté de dire». Je me limiterai au commentaire de quelques énoncés représentatifs des lieux discursifs de l'émergence de prises de position évaluatives et des procédures rhétoriques qui y sont observables. L'éthique du discours perelmanienne y contribue à une compréhension approfondie de la rhétorique argumentative et figurale mise en œuvre. Je rendrai donc compte de l'intrication discursive de la prise en charge du vrai et du bien, intrication démentant les thèses qui les soumettent à une dissociation dichotomique, et j'analyserai la mise en cause par Acrimed d'oscillations binaires et de leur caractère statique incitant à l'inaction. Je rendrai enfin également compte de l'argumentativité de l'acte de nommer, appréhendée au prisme d'opérations de recadrage métadiscursives ayant la justification d'un point de vue critique pour enjeu. La rationalité de l'argumentaire d'Acrimed est construite progressivement par son caractère explicite et par une adresse directe et constante à l'auditoire invité à juger de la pertinence d'explications, de justifications et de jugements de valeur en interaction. La thèse de l'unification des deux registres de rationalité dans l' argumentaire du sujet du discours est ainsi confirmée et illustrée par le passage suivant :

«Dans son dernier numéro (été 2009, n²1), la revue Médias [1] propose - en collaboration avec HEC [2] - un "manifeste pour la liberté de dire" sous la forme de débats entre des "personnalités de tous bords". Un tel appel ne pouvait que retenir notre attention. Pourtant, à la lecture, cette liberté semble se réduire à celle du "renard libre dans le poulailler libre", c'est-à-dire à la liberté des journalistes dominants de s'exprimer, comme ils l'entendent, selon le principe simple : fort avec le faible, faible avec le fort. Rien à voir, en somme, avec une critique radicale des médias et une lutte conséquente pour une presse indépendante et pluraliste. » 
24 libre dans le poulailler libre" » se situe dans le registre de la véridiction, mais celui-ci est en interaction avec le registre du jugement de valeur péjoratif inhérent au verbe «se réduire ». Le sens de ce verbe est explicité par son complément : la formule polémique le " "renard libre dans le poulailler libre" ». Celle-ci fait appel à la mémoire discursive collective (Paveau, 2013: 171-200) et dénonceun avatar fallacieux du libéralisme. L'intrication de ces deux types de prise en charge se manifeste sous une autre forme dans les énoncés suivants : "Cette liberté semble se réduire à celle du "renard libre dans le poulailler libre", c'est-à-dire à la liberté des journalistes dominants de s'exprimer, comme ils l'entendent, selon le principe simple: fort avec le faible, faible avec le fort ». La paraphrase explicative annoncée par «c'est-à-dire » se situe dans le registre du parler vrai démasquant une conception, jugée fallacieuse de la liberté d'expression. Cette paraphrase comprend, comme dans l'énoncé précédent, l'intrication d'un jugement de vérité et de valeur disqualifiant sa cible ( fort avec le faible, faible avec le fort »). Ce type d'hétérogénéité discursive et argumentative est activé de façon claire et naturelle par l'auteur de l'analyse. Une théorie du langage qui ne prendrait en compte que la prise en charge du vrai référentiel se condamnerait à priori à proposer une description incomplète de ces énoncés.

Le chiasme qui agence l'antithèse entre « fort » et «faible » est l'une des parties visibles de l'iceberg - soit d'une oscillation binaire à visages multiples à la fois décrite (jugement de fait) et stigmatisée (jugement de valeur) dans l'ensemble de l'article. Il s'agit pour l'auteur, U. Palheta, d'explorer les avatars d'un couple notionnel philosophique essentiel : le couple apparence/réalité soit, en l'occurrence : défense de «la liberté de dire » soit de l'acte de briser les tabous versus injonction coercitive à s'aligner sur le point de vue des hommes de pouvoir et des médias qui les soutiennent et incitent ainsi au maintien du statu quo (Koren, 1996, 2001) tout en arborant des apparences contestataires. U. Palheta dénonce une série de mensonges et prend donc un avatar fallacieux du faire croire vrai comme objet; sa prise de parole poursuit néanmoins simultanément un but à la fois aléthique et axiologique. Les autres versions de cette oscillation binaire pseudoimpartiale sont mises en mots par les énoncés suivants : «il leur plaît d'alterner l'appel à "briser les tabous" et le rappel au "bon sens", la critique de l"'idéologie dominante" (bien entendu de gauche) et l'affirmation du cela-va-de-soi (évidemment de droite). Il serait pourtant peu judicieux d'y voir une contradiction, car ce n'est là qu'un va-et-vient entre deux usages différents d'une même force, de deux formes que prend la vulgate médiatique " (je souligne). Ce mouvement de balancier valorise essentiellement l'aptitude à « cultiver son ambiguïté " (Koren, 2001) au détriment de celle qualifiée de "savoir dire non", respectivement représentatives de la sacralisation de la suspension du jugement et de son contraire : la prise de position éthique. La « vraie » « liberté de dire » ne saurait donc être confondue avec la «stratégie rhétorique» fallacieuse à laquelle recourt la revue critiquée: inverser la réalité et les apparences, "faire apparaître les rapports de domination la tête en bas ». Cette stratégie présenterait les "pauvres », les «femmes », les «noirs », les « Arabes », les « Juifs », les « homosexuels », les « handicapés » soient les « dominés » d'hier comme les nouveaux « dominants » faisant subir leurs «foudres » aux « riches », aux» hommes », aux "blancs», aux « hétérosexuels», aux « bien-portants ». Bref, le travail critique de recadrage de l'acte de nommer « l'anticonformisme de la revue Médias » a pour but de justifier et de faire partager une procédure d'évaluation et de réfutation réellement émancipatrice, à la croisée du jugement de vérité et du jugement de 
valeur. «La critique du "politiquement correct" ", qui se donne dans la revue Médias pour un "manifeste » défendant « la liberté de dire ", aurait en fait pour enjeu à contrario de «disqualifier préventivement les mobilisations » visant à déstabiliser et à compromettre la domination du pouvoir exécutif et des discours institutionnels. Ce que la NR permet d' explorer en l'occurrence, c'est le cas de l'intrication de deux types de jugements soit de l'unification de deux régimes de rationalité différents, certes, mais tout aussi nécessaires et valides l'un que l'autre. Elle permet également de discerner sous les apparences de la défense de la liberté de pensée, les réalités idéologiques masquées de l'acte de donner à des opinions inéluctablement discutables, les apparences de l'évidence irréfutable (Koren, 2011a).

\section{De l'engagement éthique du chercheur...}

La majorité des chercheurs en sciences du langage s'oppose radicalement en France à toute forme d'engagement et sacralise le devoir de neutralité. Il fallait donc que je tente de résoudre le dilemme suivant : aller à rebours de la doxa dominante, mais sans risquer de compromettre les chances de légitimation d'un droit à la différence. Mon intention n'a jamais été de nier, ce faisant, la légitimité ni l'efficacité de la distance critique impartiale, distance heuristique sans laquelle la recherche perdrait l'une de ses raisons d'être. Il s'agit uniquement pour moi de tenter de démontrer que la neutralité n'a pas le monopole de la scientificité et qu'il est possible et parfois même nécessaire d'envisager d'autres options (Koren, 2002a, 2013b et 2013c). Renoncer dans certaines circonstances à la neutralité, ce n'est pas à mes yeux basculer automatiquement dans le militantisme ou le moralisme prescriptif, mais prôner un engagement éthique aux enjeux épistémiques. Celui-ci ouvre la voie à l'analyse et l'évaluation de la rectitude des discours et de leurs acteurs dans le cas d'argumentaires incitant à la haine, à la discrimination ou au discrédit public de l'adversaire. Il existe des corpus où le renoncement à prendre position quant à la question de l'intégrité discursive et/ou rhétorique des locuteurs conduit, à mes yeux, à renoncer à une partie du savoir les concernant.

C'est le cas de l'amalgame dont j'ai tenté à plusieurs reprises de démonter les mécanismes en dépit de points de vue scientifiques adverses qui situaient ce genre de recherche hors du domaine des sciences du langage. La justification de ce positionnement est la suivante : l'analyse de l'amalgame implique que l'on recoure à la notion normative de fallacy (Koren, 1995, 1996, 2012 ; Amossy, Koren, 2010b) et donc à l' évaluation axiologique du corpus analysé. Un scientifique digne de ce nom n'aurait pas le droit de confondre les rôles d'observateur et d'acteur. La seule solution épistémique valide aux yeux d'un chercheur tenant à rester neutre consiste, dans le cas de l'amalgame, à renoncer à démontrer, par exemple, comment l'analogie, type d'argument heuristique soulignant un rapport similaire de type $\mathrm{A}$ est à $\mathrm{B}$ ce que $\mathrm{C}$ est à $\mathrm{D}$, se transforme, du fait d'une assimilation fallacieuse $A B=C D$, en assimilation totale présentée comme une évidence irréfutable. Le chercheur observateur se contentera, en l'occurrence, de décrire un échange polémique entre deux adversaires, où le recours à l'«amalgame" permet à l'un des actants de disqualifier son interlocuteur, suscitant de ce fait la révolte de sa cible. Ce type de protestation profane constitue alors l'attestation de la nature fallacieuse de l'amalgame, sans que le chercheur ait eu à se prononcer. La mauvaise foi argumentative n'est pas, à mes yeux, un mirage ni une simple question de gout ou de réception; les conséquences de l' énonciation d'un amalgame risquent, par exemple, dans le cas du terrorisme et des 
conflits armés, de légitimer à priori la destruction physique de la cible. L'amalgame avec l'un des parangons actuels du mal - soit l'acte de nomination consistant par exemple à qualifier un adversaire politique de fasciste, de populiste ou de nazi - peut masquer une incitation mortifère à la détestation. Il est donc nécessaire et légitime, dans ce cas, à mes yeux, de problématiser la question de la responsabilité énonciative (Koren, 1996, 2006a) et des conséquences des qualifications énoncées dans les espaces publics. Il me semble nécessaire, pour des raisons épistémiques et éthiques, que le chercheur remplisse ici les fonctions d'analyste et d'acteur.

L'engagement éthique du chercheur, tel que mes pérégrinations m'ont conduite à l'élaborer, doit à la conception perelmanienne de la justification les deux convictions suivantes: 1) un jugement de valeur justifié explicitement et soumis à l'évaluation critique de l'auditoire ne peut pas être prescriptif puisque ce dernier est libre, dans le champ du vraisemblable, du raisonnable et du préférable, de ne pas s'aligner sur le point de vue du proposant ; 2) la rationalité discursive axiologique existe, elle a ce type de justification, de réflexivité et d'interdépendance pour condition de possibilité. Cette conception de l'engagement éthique implique cependant que le chercheur veille à dire explicitement quand il passe de la description à l'acte d'évaluer la rectitude de son objet et quels sont les savoirs linguistiques, discursifs et argumentatifs avérés qu'il active à cette fin.

Il pourra ainsi construire un savoir qui rende compte et de la prise en charge du vrai et de celle du juste, de l'intégrité discursive comme de l'intégrité rhétorique sans porter atteinte à la liberté de juger de ses pairs et de son auditoire. Si le vrai est une évidence à accepter telle quelle, le faire croire vrai et le juste ou le bien en interaction dans le système argumentatif $\mathrm{du}$ langage et $\mathrm{du}$ discours exigent un travail sysiphique intersubjectif dont les fruits ne sont jamais définitifs ni ne peuvent être imposés à qui que ce soit.

\section{BIBLIOGRAPHIE}

AMOSSY, R. \& KOREN, R. (2010a) : «Argumentation et discours politique », Mots, 94, p. 13-21.

- (2010b) : «La "diabolisation" : un avatar du discours polémique au prisme des Présidentielles de 2007 », Au corps du texte. Mélanges en l'honneur de Georges Molinié ,Paris, H. Champion, p. 219-236.

ARENDT, H. (2005 [1964]) : Responsabilité et jugement, trad. de l'américain par J.-L. Fidel, Paris, Payot.

BOUDON, R. (1995) : Le juste et le vrai. Études sur l'objectivité des valeurs et de la connaissance, Paris, Fayard.

Charaudeau, P. \& maingueneau, D. (2002) : «Énonciateur », Dictionnaire d'analyse du discours, Paris, Éd. Le Seuil, p. 224-228.

DESCLÉS, J.-P. (2009) : « Prise en charge, engagement et désengagement », Langue française, 162, p. 29-53. 
DOURY, M. (2013) : « Positionnement descriptif, positionnement normatif, positionnement militant », Argumentation et Analyse du discours, 11 (En ligne : http://aad.revues.org/1540, consulté le $17 / 07 / 14)$.

DUCROT, O. (1972) : Dire et ne pas dire, Paris, Hermann.

EGGS, E. (1999) : « Ethos aristotélicien, conviction et pragmatique moderne », in : R. Amossy (éd.), Images de soi dans le discours, Lausanne, Delachaux et Niestlé, p. 31-59.

JAMET, D. (2008) : «I have a dream »: ces discours qui ont changé le monde, Paris, Archipoche.

KERBRAT-ORECCHIONI, C. (1980) : L'énonciation : de la subjectivité dans le langage, Paris, A. Colin.

- (1981) : « Argumentation et mauvaise foi », L'argumentation, Lyon, Presses universitaires de Lyon, p. 41-63.

- (1986): L'implicite, Paris, A. Colin

- (2005) : Le discours en interaction, Paris, A. Colin.

KOREN, R. (1991) : «Violence verbale et argumentation dans la presse révolutionnaire et contrerévolutionnaire », in : H. Chisick, I. Zinguer \& O. Elyada (eds), The Press in the French Revolution. Papers prepared for the conference «Presse d'élite, presse populaire et propagande pendant la Révolution française ", Oxford, The Voltaire Foundation, p. 319-334.

- (1993) : «Perelman et l'objectivité discursive », in: G. Haarscher (éd.), Chaïm Perelman et la pensée contemporaine, Bruxelles, Bruylant, p. 469-487.

- (1995) «Concerning an "Argumentative Monster" : The Perverted Analogy in French Journalistic Discourse », in : F. H. van Eemeren, R. Grootendorst, J. A. Blair \& C. A. Willard (éds), Proceedings of the Third Conference on Argumentation, vol. III, Reconstruction and Application, Amsterdam, International Center for the Study of Argumentation, p. 543-552.

- (1996) : Les enjeux éthiques de l'écriture de presse et la mise en mots du terrorisme, Paris, Éd. L'Harmattan.

- (1997a) : «Remarques à propos d'un groupe nominal : le miroir virulent », Scolia, 10, p. 161-182.

- (1997b) : «L'argumentaire de l'Un dans le regard de l'Autre : le jeu des dénominations », Mots, 50, p. 99-117.

- (1998) : «Transparence ", in : P. Fiala \& P. Lafon (éds), Des mots en liberté. Mélanges offerts à Maurice Tournier, Lyon, Fontenay-Saint-Cloud, ENS Éd, p. 479-488.

- (2001) : «Quand informer, c'est dire le blanc et le noir : quelques effets pervers de l'oscillation binaire », in : P. Bogaards, J. Rooryck, P. J. Smith \& V. van Gelderen (éds), Quitte ou double sens. Articles sur l'ambiguité offerts à Ronald Landheer, Amsterdam, Rodopi, p. 177-200.

- (2002a) : «La "nouvelle rhétorique" : "technique" et/ou "éthique" du discours : le cas de l'engagement du chercheur ", in : R. Koren \& R. Amossy (éds), Après Perelman : quelles politiques pour les nouvelles rhétoriques, Paris, Éd. L'Harmattan, p. 197-228.

- (2002b) : «Introduction », in : R. Koren \& R. Amossy (éds), Après Perelman : quelles politiques pour les nouvelles rhétoriques, Paris, Éd. L'Harmattan, p. 9-21.

- (2003a) : «L'engagement de l'Un dans le regard de l'Autre : le point de vue d'une linguiste », Questions de communication, 4, p. 271-277.

- (2003b) : «Contribution à l'étude des enjeux de la rhétorique laconique : le cas des indications chiffrées ", Topique, 83/2, p. 111-124.

- (2004a) : «Stratégies et enjeux de la dépolitisation par le langage dans un corpus de presse actuel », Recherches en communication, 20, p. 65-84.

- (2004b) : «Argumentation, enjeux et pratique de l'“engagement neutre" : le cas de l'écriture de presse », Semen, 17 , p. 19-40.

- (2005) : «Contribution à la régulation argumentative du différend politique : le flou polysémique du concept de "terrorisme" est-il insoluble? », in : P. Marillaud \& R. Gauthier (éds), 
Les rhétoriques politiques, Toulouse, CALS/CPST, Presses universitaires du Mirail, p. 255-270. - (2006a) : «La responsabilité des Uns dans le regard des Autres : l'effacement énonciatif au prisme de la prise de position argumentative », Semen, 22, p. 93-108.

-(2006b) : «Quels risques pour quelles prises de position "normatives" ? , Questions de communication ,9, p. 195-205.

- (2008a) « "Éthique de conviction" et/ou “éthique de responsabilité'. Tenants et aboutissants du concept de responsabilité collective dans le discours de trois quotidiens nationaux français ", Questions de communication, 13, p. 25-45.

- (2008b) : «L'analyse du discours au prisme d'un questionnement éthique », Filologia e

Lingüística Portuguesa, 9, p. 251-278.

- (2008c) : « Pour une éthique du discours : prise de position et rationalité axiologique », Argumentation et Analyse du discours, 1 (en ligne : http://aad.revues.org/263).

- (2008d) : «Prises de position "normatives" et risques », in : B. Fleury \& J. Walter (éds), Les médias et le conflit israélo-palestinien. Feux et contre-feux de la critique, Metz, Presses de l'Université Paul Verlaine-Metz, p. 83-95.

- (2009a) : «Can Perelman's NR be viewed as an ethics of discourse? ", Argumentation, vol. 23, p. 421-431.

- (2009b [2007]) : « Le récit de chiffres : enjeux argumentatifs de la "narrativisation" des chiffres dans un corpus de presse contemporain ", A contrario, 12, vol. 2, p. 66-84.

- 2010a. " "Littérature d'aveu" et prise en charge des valeurs dans Ennemis publics de Michel Houellebecq et Bernard-Henri Lévy », Argumentation et Analyse du discours, 5 (en ligne : http:// aad.revues.org/1002).

- (2010b) : «Quand l'interdisciplinarité est "état d'esprit" critique et heuristique », Questions de communication, 18, p. 159-170.

- (2011a) : «De la rationalité et \ou de l'irrationalité des polémiqueurs : certitudes et incertitudes ", Semen, 31, p. 81-95.

- (2011b) : «On axiological rationality », in : J. T. Gage (ed.), The Promise of Reason Studies in The New Rhetoric, Carbondale, Southern Illinois University Press, p. 134-144.

- (2011c) : « La logique des valeurs selon Perelman et sa contribution à l'analyse du discours », in : J. Guilhaumou \& P. Schepens (éds), Matériaux philosophiques pour l'analyse du discours, Besançon, Presses universitaires de Franche-Comté, p. 175-198.

- (2012) : «Langage et justification implicite de la violence : le cas de l' "amalgame" ", in : L. Aubry \& B. Turpin (éds), Victor Klemperer. Repenser le langage totalitaire, Paris, CNRS Éd., p. 93-105. - (2013a) : « La critique des "anciens médias" "mise au Net" : un nouveau type d'argumentation politique ? ", Argumentation et Analyse du discours, 10 (en ligne : http://aad.revues.org/1463). - (2013b) : «Introduction », Argumentation et Analyse du discours, 11, (en ligne : http:// aad.revues.org/1571).

- (2013c) : « Ni normatif ni militant : le cas de l'engagement éthique du chercheur », Argumentation et Analyse du discours, 11 (en ligne : http://aad.revues.org/1572).

- (2013d) : « Pour une éthique du discours : la prise en charge du vrai et du juste », in : C. Guérin, G. Siouffi \& S. Sorlin (éds), Le rapport éthique au discours, Berne, P. Lang, p. 39-63.

- (2015) : «Une instance à la croisée du discours et de l'éthique : le "surdestinataire" », in : J. Angermuller \& G. Philippe (éds), Analyse du discours et dispositifs d'énonciation. Autour des travaux de Dominique Maingueneau, Limoges, Lambert-Lucas, p. 137-145.

- (à paraître) : « Contraintes et autonomie : de l'identité des sujets du discours et de l'argumentation ", in : Renaissances de la rhétorique. Perelman aujourd'hui, Montréal, Nota Bene.

LAKOFF, G. \& JOHNSON, M. (1985 [1980]) : Les métaphores dans la vie quotidienne, trad. de l'américain, Paris, Éd. de Minuit. 
MAINGUENEAU, D. (1999) : « Ethos, scénographie, incorporation », in : R. Amossy (dir.), Images de soi dans le discours, Lausanne, Delachaux et Niestlé, p. 103-128.

PALHETA, U. (2009) : «Fort avec le faible, faible avec le fort : l'anticonformisme de la revue Médias ", Acrimed, 4 aout (en ligne : http://www.acrimed.org/article3191.html\#, consulté le 25/03/15).

PAVEAU, M.-A. (2013) : Langage et Morale. Une éthique des vertus discursives, Limoges, Lambert-Lucas. PERELMAN, C. \& OlBRECHTS-TYTECA, L. (1983 [1970]), Traité de l'argumentation. La nouvelle rhétorique, Bruxelles, Éd. de l'université de Bruxelles.

PERELMAN, C. (1989) : Rhétoriques, Bruxelles, Éd. de l'université de Bruxelles.

POLIN, R. (1977) La création des valeurs, $3^{e}$ éd., Paris, Vrin.

REBOUL, O. (1980) : Langage et idéologie, Paris, Presses universitaires de France.

RIBEIRO, H. J. (2012) : « Rhétorique et philosophie : Perelman et la philosophie analytique », in : B. Frydman \& M. Meyer (dirs), Chaïm Perelman (1912-2012), Paris, Puf, p. 161-185.

RORTY, R. (1990 [1979]) : L’Homme spéculaire, trad. de l'anglais, Paris, Éd. Le Seuil.

WEBER, M. (1963 [1919]) : Le savant et le politique, trad. de l'allemand par J. Freund, Paris, Plon.

\section{NOTES}

1. Contrairement à ce qui me semble essentiel : passer systématiquement de la théorie à la pratique, j'ai donné ici la primauté à la présentation et à la justification d'un parcours épistémique théorique. Le lecteur trouvera cependant tout au long de l'article des références à des travaux où sont analysés de nombreux exemples, mais aussi l'analyse plus détaillée d'un exemple type.

2. Voir également R. Rorty (1990 : 181) : « connaître la vérité d'une proposition n'est rien d'autre qu'être déterminé causalement par un objet à faire quelque chose. L'objet auquel se rapporte la proposition impose la vérité de la proposition "; on y "croirait parce que son objet nous "tient"; "des vérités nécessaires aussi paradigmatiques que le sont les axiomes de la géométrie sont censées pouvoir se passer de justifications, arguments, ou de discussions ».

3. Il existe une convergence frappante entre la description que G. Lakoff et M. Johnson (1985 : 197-199) donnent du «mythe de l'objectivisme » et l'analyse perelmanienne d'une rhétorique pseudo-objective.

4. Voir également au sujet de deux autres effets d'objectivité notoires auxquels l'écriture de presse recourt fréquemment - la citation et la syntaxe paratactique - C. Perelman \& L. OlbrechtsTyteca (ibid. : 427, 213).

5. Voir C. Perelman (1989: 466) : L'argumentation "se lie aussi à une éthique »; "dans une argumentation, il tient à nous de peser avec la plus entière bonne foi les raisons pour et les raisons contre, et, surtout, de nous faire de l'auditoire universel une idée aussi claire, aussi riche, aussi nuancée que le permet le moment où nous vivons ".

6. Le lecteur désireux d'explorer ce passage de la théorie à la pratique dans une quantité de travaux plus importante pourra les consulter dans les six catégories suivantes : 1) tenants et aboutissants de la " prise en charge du vrai et du juste ou du bien » et donc de la responsabilité énonciative du sujet qui prend position (Koren, 1997b, 2006a, 2008a, 2010a, 2011a, 2013d) ; 2) la question de l'objectivité discursive (Koren, 1993,1996, 1997a, 1998, 2001, 2002a, 2003b, 2004b, 2009 ) ; 3) la négociation de valeurs dans les discours journalistiques ou politiques (Koren, 1991, 2005, 2004a, 2013a ; Amossy, Koren, 2010a) ; 4) violence verbale et argumentation (Koren, 1991, 
1995, 1996, 2012 ; Amossy, Koren, 2010b) ; 5) lecture critique de la NR perelmanienne (Koren, 2002c, 2002a, 2011c, 2011a, 2009a, 2010b) ; 6) l'engagement éthique du chercheur (Koren, 2002a, 2003a, 2008d, 2013c).

\section{RÉSUMÉS}

Cette contribution a pour objet la reconstitution d'un parcours épistémologique personnel. Il s'agit d'y présenter et d'y justifier les pérégrinations d'une linguiste, analyste du discours et de l'argumentation, en territoire éthique. Et par territoire éthique on entendra espace de délibération interactif où le sujet et son interlocuteur s'interrogent sur des questions existentielles, sur ce qui fait sens pour eux, et tentent de vérifier la rationalité et la légitimité de leurs dires respectifs au prisme du regard critique de l'Autre. Ce parcours a pour origine un sentiment de manque dû au silence de la plupart des chercheurs en sciences du langage, en France, sur la dimension axiologique des discours sociaux et sur une fonction fondamentale de nos prises de parole : évaluer, juger, justifier nos prises de position et nos décisions. Ce silence a suscité un désir personnel de contestation ayant pour fin de revaloriser le jugement de valeur. Il s'agit de montrer que l'argumentation de valeurs autres que la vérité référentielle peut remplir une fonction tout aussi fondamentale et rationnelle que le raisonnement more geometrico. Le langage ne médiatise-t-il que des informations établissant des évidences ? Ne sert-il qu'à gérer des rapports de domination et d'influence? Seul compterait, éventuellement, dans une perspective pragmatique, le "commitment to truth", ce qui ne résout pas la question de la responsabilité énonciative du sujet énonçant explicitement ou implicitement des jugements sans rapport avec le couple notionnel vrai/faux. Les étapes successives des pérégrinations analysées dans cette contribution seront donc les suivantes : évocation de rencontres disciplinaires ou interdisciplinaires avec des travaux de recherche, à rebours des doxas objectivistes (travaux de $\mathrm{C}$. Kerbrat-Orecchionni, de Ch. Perelman, d'O. Reboul, de R. Boudon, etc.). Ces travaux m'ont permis de penser la dimension axiologique du langage et de formuler des hypothèses explicatives sur la sacralisation du vrai et la méfiance frileuse à l'égard de tout ce qui touche de près ou de loin à la mise en mots de valeurs autres que le vrai ; je problématiserai ensuite la question de l'objectivité, mais aussi de la responsabilité dans le langage et j'explorerai l'intrication de la prise en charge du vrai et du juste ou du bien dans le discours et donc de l'unification de deux régimes de rationalité parfaitement compatibles dans le système du langage. Je passerai ensuite de la théorie à la pratique afin de prouver l'existence de cette intrication dans la trame d'un texte publié sur le net. Ce parcours s'achèvera néanmoins par la brève évocation d'une dernière pérégrination consacrée à l'éthique du chercheur en sciences du langage et à la mise en question du dogme de la neutralité : si le chercheur est inéluctablement comme tout locuteur un sujet d'énonciation, peut-il et doit-il renoncer à toute forme de prise de position quel que soit son objet? Un engagement éthique épistémique est-il impossible?

The object of this contribution is the reconstitution of a personal epistemological journey: it deals with the peregrinations of a Discourse and Argumentation Analyst in the field of ethics. By ethics, I mean an interactive space of deliberation where speakers discuss existential questions and try to define what makes sense for them while attempting to verify the rationality and the legitimacy of their respective points of view through the other's critical approach. This scientific research is grounded in the perception of an epistemic gap in the publications of most French 
linguistic theories concerning the axiological dimension of social discourses. They do not inquire into a fundamental function of our speech acts: evaluation, judgment and justification of our stances and decisions. Thus the desire and necessity to revisit value judgments and demonstrate that they can be as essential and rational as the more geometrico reasoning. Does language only pass on information establishing self-evidences? Is it only a means of domination and influence? The pragmatic rule of "commitment to truth" does not solve the case of the subject's accountability for value judgments which have nothing to do with the notions of true or false. The presentation and justification of these peregrinations will follow the following steps: evocation of the interdisciplinary frame in which I have grounded the epistemic answers to my questions about the axiologization of discourse, the objectivist sacralization of truth and the distrust towards the act of taking a stance (C. Kerbrat-Orecchioni (linguist), C. Perelman (philosopher of law and rhetorician), Olivier Reboul (philosopher and rhetorician), R. Boudon (sociologist), etc.); problematization of the questions of objectivity, responsibility in language, exploration of the discursive entanglement of judgments of fact and value judgments and therefore of the unification of two regimes of rationality perfectly compatible in the system of language; illustration of the later by a case study (discursive and argumentative analysis of a text published on the Net). The ethics of the discourse analyst will be the last step of this epistemic itinerary which intends to briefly question the duty of neutrality: if the researcher is, as each speaker, a subject communicating with his audiences through language, then how can he totally neutralize his subjectivity? Does he have to remain neutral in front of any text whatsoever? Is an epistemic ethical commitment impossible?

\section{INDEX}

Mots-clés : éthique, prise en charge, sciences du langage en France, logique des valeurs, jugement de valeur, rationalité, engagement

Keywords : ethics, accountability, sciences of language in France, logic of values, value judgment, rationality, commitment

\section{AUTEUR}

\section{ROSELYNE KOREN}

ADARR, Université Bar-Ilan 